This item was submitted to Loughborough's Research Repository by the author.

Items in Figshare are protected by copyright, with all rights reserved, unless otherwise indicated.

\title{
An enriched RWG basis for enforcing global current conservation in EM modelling of capacitance extraction
}

PLEASE CITE THE PUBLISHED VERSION

https://doi.org/10.1109/ICEAA.2017.8065531

PUBLISHER

(C) IEEE

VERSION

AM (Accepted Manuscript)

LICENCE

CC BY-NC-ND 4.0

\section{REPOSITORY RECORD}

Lasisi, S.O., K. Cools, T.M. Benson, G. Gradoni, and M.T. Greenaway. 2019. "An Enriched RWG Basis for Enforcing Global Current Conservation in EM Modelling of Capacitance Extraction”. figshare.

https://hdl.handle.net/2134/27187. 


\title{
An Enriched RWG Basis for Enforcing Global Current Conservation in EM Modelling of Capacitance Extraction
}

\author{
S. O. Lasisi* \\ K. Cools \\ T. M. Benson $\ddagger$ \\ G. Gradoni ${ }^{\S}$ \\ M. T. Greenaway
}

\begin{abstract}
The RWG basis widely used in the discretisation of the EFIE is traditionally defined over interior edges of the structure's mesh when modelling charge-neutral scattering problems. In this paper, a careful extension to the RWG function space that allows for flow of current in and out of a structure via its boundary edges while maintaining current conservation is constructed. This space is appropriate for the computation of capacitance, where (i) a constant voltage difference is maintained between two components making up the device, and (ii) the total current is conserved. Using this basis implicitly enforces a constant potential difference between the metal plates (cf. natural boundary conditions), and explicitly places a restriction on the current (cf. essential boundary conditions). It improves upon the classic delta gap excitation of capacitive structures in that it is asymptotically independent of the triangulation used to describe the device's geometry.
\end{abstract}

\section{INTRODUCTION}

Electromagnetic scattering problems involving thin metal surfaces can be modelled and solved using the boundary element method (BEM). The BEM is based on the discretisation of a boundary integral equation using a set of basis functions that span the space of candidate solutions [1]. The RaoWilton-Glisson (RWG) triangular basis functions which are most often used for these purposes are typically only assigned over internal edges shared by adjacent triangles of the structure's mesh [2]. In order to allow for flow of current in and out of the structure via its boundary edges, as can be found in edge-fed voltage excitations, half RWGs (one triangle out of the pair of triangles in a RWG basis)

\footnotetext{
*George Green Institute for Electromagnetic Research, University of Nottingham, Nottingham NG7 2RD, United Kingdom, e-mail: shakirudeen.lasisi@nottingham.ac.uk.

${ }^{\dagger}$ George Green Institute for Electromagnetic Research, University of Nottingham, Nottingham NG7 2RD, United Kingdom, e-mail: kristof.cools@nottingham.ac.uk.

${ }^{\ddagger}$ George Green Institute for Electromagnetic Research, University of Nottingham, Nottingham NG7 2RD, United Kingdom, e-mail: trevor.benson@nottingham.ac.uk.

§School of Mathematical Sciences \& George Green Institute for Electromagnetic Research, University of Nottingham, Nottingham NG7 2RD, United Kingdom, e-mail: gabriele.gradoni@nottingham.ac.uk.

IDepartment of Physics, Loughborough University, Loughborough LE11 3TU, United Kingdom, e-mail: m.t.greenaway@lboro.ac.uk.
}

need to be assigned to boundary edges coinciding with the ports of the device. In [3], a preconditioned BEM was introduced for the scattering problem where the voltage was described at both ports. In most usage scenarios only the difference between the potential at the two ports is relevant. In addition, even though current is allowed to flow from one component into another, the total current in the system is conserved and in particular the sum of the charge accumulated on the two plates is zero. A finite element space that can be used for the solution of this scattering problem should be rich enough to allow currents to flow from one sheet to the other (without specifying the absolute value of the scalar potential), but constraint enough to enforce current conservation (the net current flowing out one port needs to flow back in through the sibling port).

This finite element space was constructed with the aim to develop a computational electromagnetic model (EM) of a resonant tunnelling diode (RTD) used as high frequency oscillators in the terahertz range. In this configuration a bias voltage $V$ is applied at the terminals of two overlapping structures separated by a tunnel barrier (see Ref. [4]). This voltage induces charge on each structure and a tunnel current through the barrier. The enriched RWG works not only as a suitable feed model but also a load model that supports the inclusion of the nonlinear negative differential conductance (NDC) behaviour that is realized in the operation of the diode. Other feed and load models found in literature include the delta gap, magnetic frill generator and its approximations [5] - [6].

This paper discusses the formulation of an enriched RWG function. It is demonstrated in the solution of a capacitor extraction problem, where a constant voltage difference is applied at the terminals of the capacitor plates. The current induced on the plates of the capacitor is calculated with the BEM and used in the computation of the capacitance. 


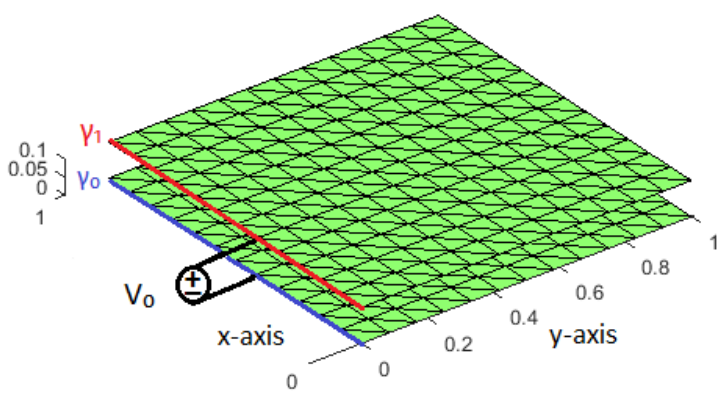

Figure 1: Voltage excited Capacitor plates

\section{FORMULATION}

Consider a capacitor modelled by the surface $\Gamma=$ $\Gamma_{1} \cup \Gamma_{0}$ comprising two components (Fig. 1). The total electric field $\boldsymbol{e}=\boldsymbol{e}^{s}$ (no incident field is considered in this application) is such that its tangential components vanishes on $\Gamma$ :

$$
\hat{\boldsymbol{n}} \times e^{s}(\boldsymbol{j})=0
$$

The scattered field can be computed from the induced current through the application of the single layer operator:

$$
\begin{aligned}
T \boldsymbol{j}= & T_{h} \boldsymbol{j}+T_{s} \boldsymbol{j} \\
=\hat{\boldsymbol{n}} & \times \frac{1}{i k} \eta \operatorname{grad} \int_{\Gamma} G\left(\boldsymbol{r}, \boldsymbol{r}^{\prime}\right) \operatorname{div} \boldsymbol{j}\left(\boldsymbol{r}^{\prime}\right) d S^{\prime} \\
& \quad-\hat{\boldsymbol{n}} \times i k \eta \int_{\Gamma} G\left(\boldsymbol{r}, \boldsymbol{r}^{\prime}\right) \boldsymbol{j}\left(\boldsymbol{r}^{\prime}\right) d S^{\prime}=0
\end{aligned}
$$

Applying a test function $\hat{\boldsymbol{n}} \times \boldsymbol{k}$ and integrating by parts yields the following equation written in terms of the voltages at the ports $\gamma_{0}$ and $\gamma_{1}$ where $\hat{\boldsymbol{m}}$ is the vector tangential to $\Gamma$ and normal to the edges

$$
\begin{aligned}
t_{h}(\boldsymbol{k}, \boldsymbol{j}) & +t_{s}(\boldsymbol{k}, \boldsymbol{j}) \\
& -\left[(\hat{\boldsymbol{m}} \cdot \boldsymbol{k}, \Phi)_{\gamma_{0}}+(\hat{\boldsymbol{m}} \cdot \boldsymbol{k}, \Phi)_{\gamma_{1}}\right] \\
=- & \frac{1}{i k} \eta \int_{\Gamma} \operatorname{div} \boldsymbol{k} \int_{\Gamma} G\left(\boldsymbol{r}, \boldsymbol{r}^{\prime}\right) \operatorname{div} \boldsymbol{j}\left(\boldsymbol{r}^{\prime}\right) d S^{\prime} d S \\
& -i k \eta \int_{\Gamma} \int_{\Gamma} G\left(\boldsymbol{r}, \boldsymbol{r}^{\prime}\right) \boldsymbol{k} \cdot \boldsymbol{j}\left(\boldsymbol{r}^{\prime}\right) d S^{\prime} d S \\
- & {\left[\int_{\gamma_{0}}(\hat{\boldsymbol{m}} \cdot \boldsymbol{k}) \Phi d l+\int_{\gamma_{1}}(\hat{\boldsymbol{m}} \cdot \boldsymbol{k}) \Phi d l\right]=0 }
\end{aligned}
$$

Current conservation between the plates can be explicitly enforced by only considering candidate solutions such that the current at ports $\gamma_{0}$ and $\gamma_{1}$ satisfies the equation $I_{1}=I_{0}$ i.e.

$$
\int_{\gamma_{1}} \hat{\boldsymbol{m}} \cdot \boldsymbol{j} d l=-\int_{\gamma_{0}} \hat{\boldsymbol{m}} \cdot \boldsymbol{j} d l
$$

The space of test functions will be chosen to be $\hat{\boldsymbol{n}} \times$ the space of basis functions. In other words, for the test functions $\hat{\boldsymbol{n}} \times \boldsymbol{k}$, it also holds that

$$
\int_{\gamma_{1}} \hat{\boldsymbol{m}} \cdot \boldsymbol{k} d l=-\int_{\gamma_{0}} \hat{\boldsymbol{m}} \cdot \boldsymbol{k} d l
$$

For the solution of the scattering problem it holds that the voltages are constant along the ports, and the potential difference $\Phi_{\gamma_{1}}-\Phi_{\gamma_{0}}=V$ equals the applied voltage $V_{0}$. Equation (3) can be simplified using (5) to result in the EFIE with excitation in terms of the voltage difference $V$.

$$
t_{h}(\boldsymbol{k}, \boldsymbol{j})+t_{s}(\boldsymbol{k}, \boldsymbol{j})=(\hat{\boldsymbol{m}} \cdot \boldsymbol{k}, V)_{\gamma_{1}}
$$

\section{BASIS FUNCTION}

In this section a finite element basis is constructed such that the constraint (4) is fulfilled for all functions in the space spanned by this basis. The current is approximated by a linear expansion

$$
\boldsymbol{j}=\sum_{n=1}^{N} a_{n} \boldsymbol{f}_{m}
$$

where $\boldsymbol{f}_{m}$ are the RWG basis and $a_{n}$ are the unknown weighting coefficients.

The RWG space used in the modelling of charge neutral scattering does not include degrees of freedom on the boundary of the sheets that make up the device [2]. In this section, it is explained how the space can be enriched to allow a non-zero current density to flow into the ports of the device whilst at the same time enforcing the global current conservation constraint. This is the correct space to model the dynamics of a voltage source driven capacitance. It improves upon delta gap excitations, which are popular in this context, in that it is independent of the mesh of the structure and allows the modelling of variations in current density along the port. To include a source or feed model however requires a voltage jump and flow of current across the set of boundary edges that define the feed ports. It was explained in previous section how these sources can be incorporated and how they give rise to source terms in the linear system.

As a starting point, monopole (half) RWGs are attached to the boundary edges on $\gamma_{0}$ and $\gamma_{1}$. Next, three types of basis functions are constructed, each complying with (4):

1. Classic RWGs are associated with the internal edges of the structures mesh. 
2. For every vertex internal to the port $\gamma=$ $\gamma_{0} \cup \gamma_{1}$, we define a function that is the difference of the two half RWGs attached to the two segments on $\gamma$ adjacent to the vertex under consideration (Fig. 2). This is to account for varying current distributions and the net current on each port sums up to zero i.e

$$
\int_{\gamma_{1}} \hat{\boldsymbol{m}} \cdot \boldsymbol{f}^{v} d l=\int_{\gamma_{0}} \hat{\boldsymbol{m}} \cdot \boldsymbol{f}^{v} d l=0
$$

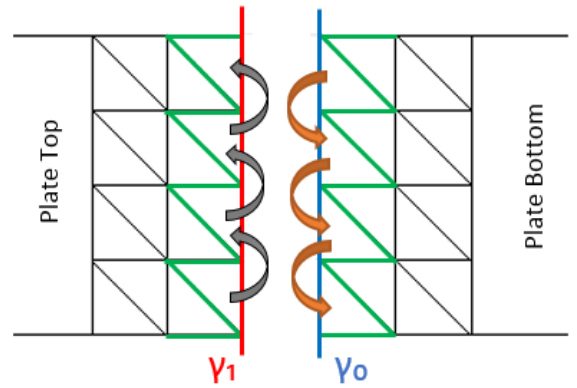

Figure 2: Diagram illustrating alternating current between adjacent edges of the same port. See condition 2 .

3. A single basis function conserving global current is constructed by adding all outward pointing half RWGs on edges of $\gamma_{1}$ and all inward pointing half RWGs on edges of $\gamma_{0}$ (Fig. $3)$. The basis is normalized such that

$$
\int_{\gamma_{1}} \hat{\boldsymbol{m}} \cdot \boldsymbol{f}^{c} d l=-\int_{\gamma_{0}} \hat{\boldsymbol{m}} \cdot \boldsymbol{f}^{c} d l=1
$$

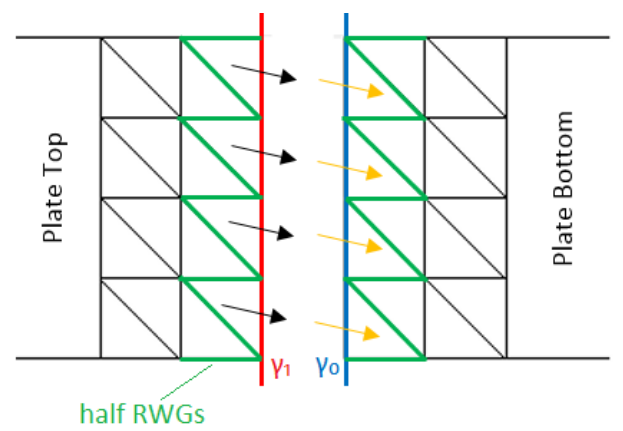

Figure 3: Schematic diagram showing current conservation between the ports on the edges of the two plates. See condition 3.

In summary, $\boldsymbol{j}$ can be written as:

$$
\boldsymbol{j}=\sum a_{m}^{i} \boldsymbol{f}^{i}+\sum a_{m}^{v} \boldsymbol{f}^{v}+a^{c} \boldsymbol{f}^{c}
$$

The superscripts i,v and c represent the classic interior RWG, varying and constant half-RWG functions.

\section{NUMERICAL RESULTS}

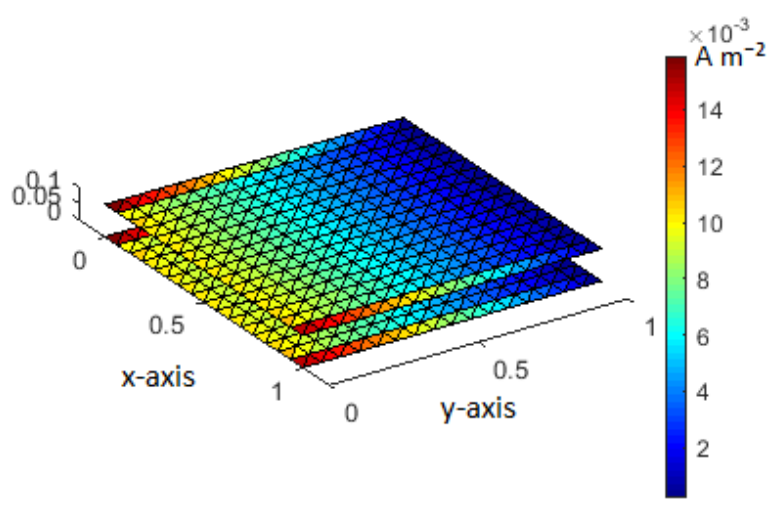

Figure 4: Plot of face currents corresponding to the solution of the BEM system

Consider the geometry in Fig 1 utilized in the previous sections. The plates are of area $1 \mathrm{~m}^{2} \mathrm{sep}-$ arated by a distance $0.1 \mathrm{~m}$ and partitioned into 1024 triangular cells (resulting in 1503 degrees of freedom). A voltage difference of $V_{0}=1 \mathrm{~V}$ is applied between the two labelled ports of the plates, thus $C=Q$. Utilizing the previously derived EFIE and enriched basis function, the simulation is carried out in the frequency domain at $15 \mathrm{MHz}$ where $\lambda=20 \mathrm{~m} \gg l=1.0 \mathrm{~m}$. Fig. 4 shows the distribution of the surface currents $j$ on the metal conductors from the BEM solution of the EFIE. Clearly current is allowed to flow from one plate into another via the ports. Fig. 5 plots the potential difference along the $\mathrm{z}$-axis of the plates computed from the induced face currents as in

$$
\Phi=-\frac{1}{i k} \eta \int_{\Gamma} G\left(\boldsymbol{r}, \boldsymbol{r}^{\prime}\right) \operatorname{div} \boldsymbol{j}\left(\boldsymbol{r}^{\prime}\right) d S^{\prime}
$$

It can be seen from Fig. 5 that $\Phi_{1}=V_{0} / 2, \Phi_{0}=$ $-V_{0} / 2$ and $\Phi \rightarrow 0$ as $z \rightarrow \pm \infty$. Similarly the total charge $Q$ is determined by

$$
Q=-\frac{1}{i \omega} \int \operatorname{div} \boldsymbol{j}\left(\boldsymbol{r}^{\prime}\right) d s^{\prime}
$$

which can be simplified given the properties of the finite element space to

$Q=-\frac{1}{i \omega} \int_{\gamma_{1}} \hat{\boldsymbol{m}} \cdot \boldsymbol{j}\left(\boldsymbol{r}^{\prime}\right) d l=-\frac{a^{c}}{i \omega} \int_{\gamma_{1}} \hat{\boldsymbol{m}} \cdot \boldsymbol{f}^{c} d l=-\frac{a^{c}}{i \omega}$

where $a^{c}$ is the coefficient of the constant half RWG.

The capacitance can be found to be $C=117$ $\mathrm{pF}$. This is the same order of magnitude as $C_{p}$ obtained using the well known parallel plate capacitance equation: $C_{p}=\epsilon_{0} \epsilon_{r} A / d \approx 88 \mathrm{pF}$. Note that 


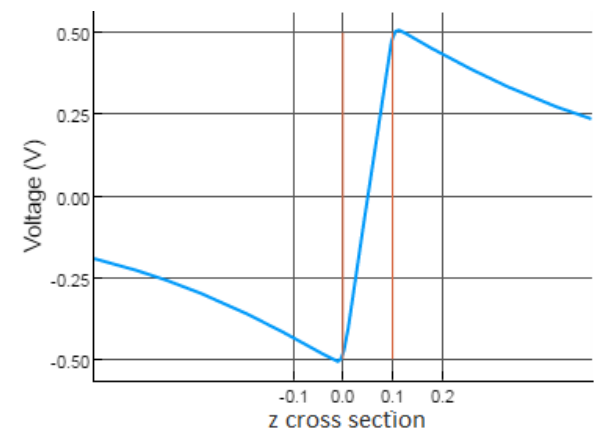

Figure 5: Plot of computed voltage along z-cross section of the plates $(x=0.5 \mathrm{~m}, y=0.5 \mathrm{~m})$

the derivation of $C_{p}$ assumes a uniform electric field between the two plates and that the field outside this region is negligible. In Fig. 6 we show the $\mathrm{z}$ component of the electric field and find that it is approximately uniform between the two plates (the $\mathrm{x}$ and $\mathrm{y}$ components of the field are approximately zero and can be ignored $E_{x}, E_{y} \approx 0$ ), and quickly goes to zero outside the boundaries of the structure. However, the fringing fields at the edges behaves so as to increase the length of the plates resulting in a higher capacitance. For capacitance in the pico-Farads range as in the example above, this along with other factors such as the order of discretisation and accuracy of the numerical solution manifests as the observable difference seen in the approximate and numerical solutions.

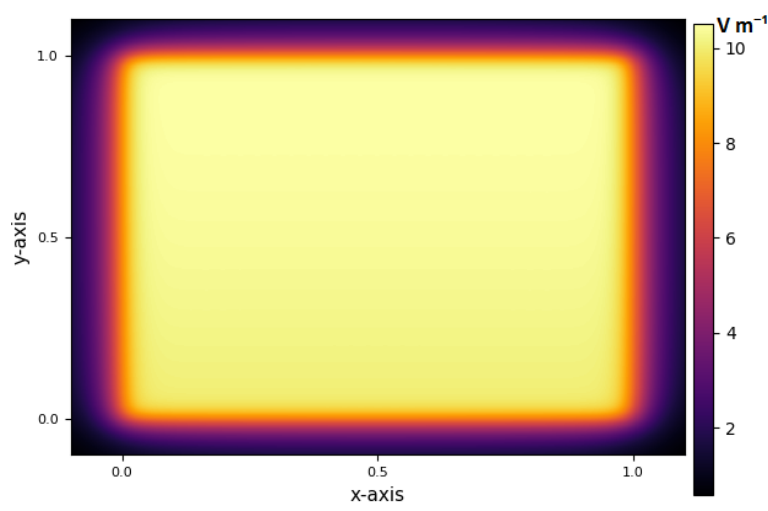

Figure 6: Plot of absolute value of z-component of electric field $\left(E_{z}\right)$ between the plates at $z=0.05 \mathrm{~m}$

\section{References}

[1] C. A. Brebbia. Boundary Element Techniques in Computer-Aided Engineering. Martinus Nijhoff Publishers, Dordrecht, 1984.

[2] S. Rao, D. Wilton, and A. Glisson. Electromagnetic scattering by surfaces of arbitrary shape.
IEEE Transactions on Antennas and Propagation, 30(3):409-418, May 1982.

[3] F. Andriulli. K. Cools. A well conditioned efie for capacitance extraction. IEEE International Symposium on Antennas and Propagation, pages 1569-1570, 2016.

[4] K. S. Novoselov A. Mishchenko A. K. Geim T. M. Fromhold J. Gaskell, L. Eaves and M. T. Greenaway. Graphene-hexagonal boron nitride resonant tunneling diodes as high-frequency oscillators. Applied Physics Letters, 107(10), September 2015.

[5] R. Maaskant and M. Arts. Reconsidering the voltage-gap source model used in moment methods. IEEE Antennas and Propagation Magazine, 52(2):120-125, April 2010.

[6] Y. H. Lo, L. J. Jiang, and W. C. Chew. Finitewidth feed and load models. IEEE Transactions on Antennas and Propagation, 61(1):281-289, Jan 2013. 